\title{
Is a perceived supportive physical environment important for self-reported leisure time physical activity among socioeconomically disadvantaged women with poor psychosocial characteristics? An observational study
}

Verity J Cleland ${ }^{1,2}$, Kylie Ball ${ }^{1 *}$ and David Crawford ${ }^{1}$

\begin{abstract}
Background: Over the past decade, studies and public health interventions that target the physical environment as an avenue for promoting physical activity have increased in number. While it appears that a supportive physical environment has a role to play in promoting physical activity, social-ecological models emphasise the importance of considering other multiple levels of influence on behaviour, including individual (e.g. self-efficacy, intentions, enjoyment) and social (e.g. social support, access to childcare) factors (psychosocial factors). However, not everyone has these physical activity-promoting psychosocial characteristics; it remains unclear what contribution the environment makes to physical activity among these groups. This study aimed to examine the association between the perceived physical environment and self-reported leisure-time physical activity (LTPA) among women living in socioeconomically disadvantaged areas demonstrating different psychosocial characteristics.

Methods: In 2007-8, 3765 women (18-45 years) randomly selected from low socioeconomic areas in Victoria, Australia, self-reported LTPA, and individual, social and physical environmental factors hypothesised within a socialecological framework to influence LTPA. Psychosocial and environment scores were created. Associations between environment scores and categories of LTPA (overall and stratified by thirds of perceived environment scores) were examined using generalised ordered logistic regression.

Results: Women with medium and high perceived environment scores had $20-38 \%$ and $44-70 \%$ greater odds respectively of achieving higher levels of LTPA than women with low environment scores. When stratified by thirds of psychosocial factor scores, these associations were largely attenuated and mostly became non-significant. However, women with the lowest psychosocial scores but medium or high environment scores had 76\% and 58\% higher odds respectively of achieving $\geq 120$ minutes/week (vs. $<120$ minutes/week) LTPA.

Conclusions: Acknowledging the cross-sectional study design, the findings suggest that a physical environment perceived to be supportive of physical activity might help women with less favourable psychosocial characteristics achieve moderate amounts of LTPA (i.e. $\geq 120$ minutes/week). This study provides further support for research and public health interventions to target perceptions of the physical environment as a key component of strategies to promote physical activity.
\end{abstract}

Keywords: Social-ecological model, Health behaviour, Socioeconomic disadvantage, Cross-sectional, Survey research

\footnotetext{
* Correspondence: kylie.ball@deakin.edu.au

${ }^{1}$ Centre for Physical Activity \& Nutrition Research, Deakin University, 221

Burwood Hwy, Burwood, Victoria 3125, Australia

Full list of author information is available at the end of the article
} 


\section{Background}

Women of childbearing age and those experiencing socioeconomic disadvantage are population groups at high risk for physical inactivity [1,2] and consequently poor health and chronic disease. In particular, women experiencing socioeconomic disadvantage are at particular risk of inadequate leisure-time physical activity, with physical activity likely to be undertaken as a matter of necessity rather than choice [3]. As such, women experiencing socioeconomic disadvantage are important targets for physical activity promotion. In order to effectively target policies and programs, an understanding of the influences on physical activity is necessary. Social-ecological models (e.g. [4,5]) provide useful frameworks for understanding drivers of health behaviours such as physical activity. These models acknowledge multiple layers of influence, and propose that a range of individual, social and physical environment factors interact to influence behaviour [4,5]. Typically Social Ecological models do not specifically characterize the relationship of the environment with the individual, and hence investigations of different models incorporating both psychosocial and environmental factors simultaneously can help further elucidate associations among factors predicting behaviours such as physical activity, and advance social ecological theories of behaviour.

Many individual and social factors (termed here 'psychosocial factors', ie encompassing both psychological and social influences) have been identified as important predictors of physical activity behaviour (e.g. self-efficacy, enjoyment, intentions, skills, childcare, social support, dog ownership [6-8]), as have environmental factors (e.g. facilities, destinations, walkability, safety, sidewalks [9]). However, many women, particularly those experiencing socioeconomic disadvantage, lack 'physical activity-promoting' individual and social characteristics. For example, a number of women do not have high levels of self-efficacy, may not enjoy physical activity, or may not have the skills to be active; they may have unsupportive family and friends, or have no access to childcare [6,7]. It is important to recognise that many women do not possess these factors, because achieving improvements in these characteristics that result in increases in physical activity can be difficult $[10,11]$. The contribution of the physical environment to physical activity amongst these women and how this compares to those with more favourable psychosocial characteristics is unknown. A better understanding of how these factors relate to physical activity may provide insights for public health intervention - that is, are there things we can do at the environmental level that may impact on physical activity behaviour, irrespective of individual- and social-level factors?

While we and others' have previously demonstrated that there are associations between individual, social and environmental factors and physical activity separately
$[8,12,13]$, no previous research has examined whether there are differential effects on physical activity when there are 'mismatches' between psychosocial- and environmental-level characteristics. The study aimed to examine the patterns of associations between perceptions of the physical environment and leisure-time physical activity (LTPA) among socioeconomically disadvantaged women stratified by different levels of psychosocial variables.

\section{Methods}

Data were collected in the Resilience for Eating and Activity Despite Inequality (READI) study during 2007-8 $[14,15]$. The Deakin University Human Research Ethics Committee approved this study, with written informed consent obtained.

\section{Participants}

Postcodes in the bottom third of the Socio-Economic Index for Areas (SEIFA) [16] distribution in Victoria, Australia, were classified as 'disadvantaged'. Neighbourhoods scoring in the bottom third of this aggregate index are characterized, for example, by higher proportions of residents with relatively low incomes, low education levels, and higher rates of unemployment. Forty urban and 40 rural areas were randomly selected. From the Australian electoral roll (compulsory from age 18), 150 women (18-45 years) were randomly identified in each area $(n=11,940$; some areas had $<150$ eligible women). After excluding 861 'return to sender' surveys, 4,938 (45\%) women completed a postal questionnaire. Excluded were those moving from the sampled suburb prior to survey completion $(n=571)$, surveys completed by a non-intended person $(n=3)$, those withdrawing data $(n=2)$, or those $<17$ - or $>46$-years old $(\mathrm{n}=13)$. Of the remaining participants $(\mathrm{n}=4,349)$, those pregnant $(\mathrm{n}=210)$; with missing data for pregnancy $(n=40)$, physical activity $(n=165)$, or environment $(\mathrm{n}=97)$; or with $>1$ missing psychosocial factor $(\mathrm{n}=22)$ or demographic factor $(\mathrm{n}=74)$ were excluded, leaving 3,765 participants.

\section{Measures}

\section{Leisure time physical activity}

Participants reported past week physical activity duration, frequency and intensity within leisure, transportation, occupational and domestic domains in the International Physical Activity Questionnaire (long version), which has demonstrated high reliability and acceptable validity across at least 12 countries [17]. Leisure time physical activity (LTPA) is discretionary and under volitional control (unlike for example, occupational activity) and therefore more likely to be amenable to intervention. In addition, LTPA conceptually is most likely to be influenced by local neighborhood environmental features (i.e., we would not 
expect to observe an association between perceived neighbourhood aesthetics and domestic activity, or between the local physical activity environment and occupational activity) [18]. For these reasons, LTPA was chosen as the focus for this paper, and included time spent walking for leisure, as well as moderate- and vigorous-intensity physical activity for leisure. Minutes/week of LTPA were calculated, then categorised because of high skewness resulting from the large number of zero values (a suitable transformation could not be determined). Domain-specific physical activity guidelines currently do not exist, so a categorical variable was calculated by classifying LTPA as 0 minutes/week (to account for the large number of zero values), then using tertile cutpoints to categorise the non-zero data as 1-119 minutes/week, 120-279 minutes/week, and $\geq 280$ minutes/week. Tertiles were selected as they allow for an acceptable number of participants in each category while retaining sensitivity.

\section{Psychosocial score}

A 'psychosocial score' was created using four individual (self-efficacy, enjoyment, intentions, behavioural skills) and four social (child care, family support, friend support, dog ownership) variables previously found to be associated with LTPA in this [8] and other [2,13,19] samples. Where scales were used, internal reliability as assessed in this sample is presented as Cronbach's alpha. Participants rated their confidence (self-efficacy) on a five-point Likert scale in being active in five difficult circumstances [20]; responses were summed (Cronbach's alpha =0.82). Responses to six items on a seven-point Likert scale about physical activity enjoyment [21] were summed (Cronbach's alpha $=0.95$ ). A seven-point Likert scale was used to assess activity intentions [22], and behavioural skills were assessed by summing two questions about past week frequency of goal setting and planning for physical activity [22] (Cronbach's alpha $=0.83$ ). Participants reported access to childcare if they wanted to be active (not applicable, yes, no), and dog ownership (yes, no). Family social support was assessed by asking how often in the past year family members engaged in physical activity with them or encouraged physical activity [23]; responses to the two questions were summed (Cronbach's alpha $=0.76$ ). The same questions were asked in reference to friends/work colleagues (Cronbach's alpha $=0.69$ ).

Childcare was scored 0 (not available) or 1 (available or not applicable), and dog ownership was scored 0 (no dog) or 1 (dog owner). To overcome differences in scales, each of the other four individual and two social variables were categorised into thirds and allocated a score of 0 (lowest third), 1 (middle third) or 2 (highest third). Scores for the eight variables were summed to create an overall 'psychosocial score, with a possible range of $0-14$. This score was classified as low (0-6), medium (7-9) or high (10-13) based on tertile cutpoints, with higher scores indicating more favourable psychosocial characteristics. While some information on specific variables may be lost, this approach provides an opportunity to categorise discordant participant psychosocial 'profiles' in a parsimonious manner. That is, a range of psychosocial variables are incorporated into a single score, providing an aggregate indicator of psychosocial support, and reducing complexity and issues associated with multiple testing of numerous constructs in isolation. The internal consistency of the psychosocial score was reasonable (Cronbach's alpha $=0.66$ ).

\section{Perceived environment score}

A perceived neighbourhood 'environment score' was created from three self-reported variables described in our earlier work [8]. Briefly, personal safety (sum of three items; Cronbach's alpha $=0.85$ ), aesthetics (sum of five items; Cronbach's alpha $=0.76$ ), and the 'physical activity environment' (sum of seven items; Cronbach's alpha = $0.80)$ were assessed on five-point Likert scales adapted from an existing measure [24]. To overcome differences in scales, each of the three environmental variables was categorised into thirds and allocated a score of 0 (lowest third), 1 (middle third) or 2 (highest third). Scores for the three variables were summed to create the score, with a possible and actual range of $0-6$. This score was classified as low (0-1), medium (2-3) or high (4-6) based on tertile cutpoints, with higher scores indicating more favourable physical environments. The internal consistency of the environment score was reasonable (Cronbach's alpha $=0.63$ ).

\section{Potential covariates}

Self-reported demographic characteristics included age; marital status (married/living as married, previously married, or not married); level of education (low = less than Year 12; mid = Year 12, certificate/trade/diploma; high = tertiary); employment status (full-time work, part-time work, or not employed); children <18 years (none, 1, 2, 3+); birth country (Australia or outside Australia); height and weight, converted to body mass index (BMI) and categorised as healthy (including underweight), overweight or obese $\left(\leq 25 \mathrm{~kg} / \mathrm{m}^{2}, 25-29.9 \mathrm{~kg} / \mathrm{m}^{2}, \geq 30 \mathrm{~kg} / \mathrm{m}^{2}\right.$, respectively) [25]; presence of illness/injury/disability likely to affect physical activity (yes, no); current smoking (yes, no); and access to a motor vehicle (yes, no). Area of residence (urban/rural) was assessed as a potential covariate. Urban and rural are defined elsewhere in detail (Cleland, Hume, et al., 2010). Briefly, urban areas included metropolitan Melbourne, rural cities (defined by the Regional Infrastructure Development Act 1999 [Vic]) and all suburbs completely within a $10 \mathrm{~km}$ radius of the centroid of these cities, and all suburbs completely within a $10 \mathrm{~km}$ radius of the centroid of other Victorian cities with a population of $\geq 20,000$. Rural areas were those falling outside of 
metropolitan Melbourne and outside of a $25 \mathrm{~km}$ radius of the rural cities.

\section{Analyses}

Demographic characteristics (potential covariates) are presented for the overall sample and across categories of LTPA. Differences across these categories were tested using one-way analysis-of-variance (equal variances) or Kruskal-Wallis equality-of-populations rank test (unequal variances) for continuous variables, and Pearson's chi-square test for categorical variables. Mean (and standard deviation) and median (and inter-quartile range) LTPA is presented overall and across categories of environment score categories (low, medium, high) and psychosocial score categories (low, medium, high). Cuzick's non-parametric test for trend was used to assess whether a significant trend was evident for LTPA across increasing environment score categories and across increasing psychosocial score categories.

Because of the ordered but not evenly spaced nature of the outcome data, generalised ordered logistic regression was employed by using the 'gologit2' command [26] in Stata, Version 10.2 (Statacorp, College Station, Texas) to calculate odds ratios (OR) and 95\% confidence intervals (CI) for increasing categories of LTPA across categories of the environment score. These analyses were repeated stratified by psychosocial score categories to examine the association between the environment and

Table 1 Socio-demographic and health characteristics of women in the READI study by LTPA

\begin{tabular}{|c|c|c|c|c|c|}
\hline & \multirow[t]{2}{*}{ Overall } & \multicolumn{4}{|l|}{ LTPA } \\
\hline & & $\begin{array}{l}0 \text { mins/week } \\
(n=1021)\end{array}$ & $\begin{array}{l}1-119 \text { mins/wk } \\
(n=762)\end{array}$ & $\begin{array}{l}120-279 \text { mins/wk } \\
(n=1034)\end{array}$ & $\begin{array}{l}\geq 280 \text { mins } / w k \\
(n=948)\end{array}$ \\
\hline Age (years), $M(S D)$ & $34.6(8.2)$ & $35.7(7.9)$ & $34.0(8.1)$ & $34.4(8.4)$ & $34.1(8.4)^{* *}$ \\
\hline BMI $\left(\mathrm{kg} / \mathrm{m}^{2}\right), M(S D)$ & $26.0(6.1)$ & $27.2(7.0)$ & $25.8(5.9)$ & $25.7(5.5)$ & $25.3(5.6)^{* *}$ \\
\hline \multicolumn{6}{|l|}{ Level of education, $n(\%)$} \\
\hline Low & $833(22.3)$ & $317(31.3)$ & $139(18.3)$ & $206(20.0)$ & $171(18.2)^{* *}$ \\
\hline Medium & $1937(51.7)$ & $520(51.3)$ & $404(53.3)$ & $510(49.5)$ & $503(53.4)$ \\
\hline High & $974(26.0)$ & $176(17.4)$ & $215(28.4)$ & $315(30.6)$ & $268(28.5)$ \\
\hline \multicolumn{6}{|l|}{ Employment status, n (\%) } \\
\hline Working full-time & $1426(38.6)$ & $361(36.1)$ & $272(36.4)$ & $392(38.4)$ & $401(43.4)^{* *}$ \\
\hline Working part-time & $1091(29.5)$ & $272(27.2)$ & $227(30.4)$ & $309(30.2)$ & $283(30.6)$ \\
\hline Not employed & 1179 (31.9) & $368(36.8)$ & $249(33.3)$ & $321(31.4)$ & $241(26.1)$ \\
\hline Access to a motor vehicle, $n(\%)$ & $3,517(93.7)$ & $948(93.0)$ & 713 (93.6) & $959(93.3)$ & $897(94.9)$ \\
\hline \multicolumn{6}{|l|}{ Marital status, $n(\%)$} \\
\hline Not married & $1000(26.6)$ & $247(24.2)$ & $184(24.2)$ & $277(26.8)$ & $292(30.9)^{*}$ \\
\hline Married/Living as married & $2443(65.0)$ & $667(65.4)$ & $528(69.3)$ & $671(65.0)$ & $577(61.0)$ \\
\hline Previously married & $317(8.4)$ & $106(10.4)$ & $50(6.6)$ & $84(8.1)$ & $77(8.1)$ \\
\hline \multicolumn{6}{|l|}{ No. of children <18 yrs, $n$ (\%) } \\
\hline None & 1464 (39.3) & $328(32.6)$ & $287(38.2)$ & $410(40.0)$ & $439(46.8)^{* *}$ \\
\hline One & $661(17.8)$ & $215(21.4)$ & $116(15.4)$ & $188(18.3)$ & $142(15.1)$ \\
\hline Two & $968(26.0)$ & $280(27.8)$ & $208(27.7)$ & $261(25.5)$ & $219(23.3)$ \\
\hline$\geq$ Three & $629(16.9)$ & $183(18.2)$ & $141(18.8)$ & $166(16.2)$ & $139(14.8)$ \\
\hline Urban area of residence, $n$ (\%) & $1735(46.1)$ & $496(48.6)$ & $341(44.8)$ & $471(45.6)$ & $427(45.0)$ \\
\hline Born in Australia, $n(\%)$ & 3371 (89.6) & $883(86.5)$ & $687(90.3)$ & $933(90.2)$ & $868(91.6)^{*}$ \\
\hline Current smoker, $n$ (\%) & $608(16.2)$ & $269(26.4)$ & $110(14.5)$ & $124(12.0)$ & $105(11.1)^{* *}$ \\
\hline Illness/injury/disability, n (\%) & $425(11.3)$ & $152(14.9)$ & $91(12.4)$ & $87(8.4)$ & $91(9.7)^{* *}$ \\
\hline \multicolumn{6}{|l|}{ Weight status, $n$ (\%) } \\
\hline Healthy weight & $1913(53.1)$ & $442(45.8)$ & $401(54.4)$ & $536(54.0)$ & $534(58.8)^{* *}$ \\
\hline Overweight & $912(25.3)$ & $238(24.7)$ & $189(25.6)$ & $271(27.3)$ & $214(23.5)$ \\
\hline Obese & 778 (21.6) & 285 (29.5) & $147(20.0)$ & 185 (18.7) & $161(17.7)$ \\
\hline
\end{tabular}

READI resilience for eating and inactivity despite inequality study; SD standard deviation; BMI body mass index; LTPA leisure time physical activity. ${ }^{* *} \mathrm{p}<0.001,{ }^{*} \mathrm{p}<0.01$ for differences across categories of LTPA from one-way analysis-of-variance or Kruskal-Wallis equality-of-populations rank test (continuous variables) or Pearson's chi-square test (categorical variables). 
LTPA across categories of psychosocial scores. In generalised ordered logistic regression, the actual values of the dependent variable (LTPA) are irrelevant except that it is assumed that larger values correspond to 'higher' outcomes. The results indicate the odds of participating in increasing LTPA associated with one-unit increases in the independent variables (e.g. the difference between a low and medium environment score). Unlike standard logistic regression where increasing categories are compared to a typical 'referent' group (e.g. the bottom/lowest category), ordered logistic regression compares each increasing outcome category to all data falling below the cutpoint for that category (e.g. $<120$ minutes/week is the referent group for calculating the odds of achieving $\geq 120$ minutes/week LTPA, while $<280$ minutes/week is the referent group for calculating the odds of achieving $\geq 280$ minutes/week LTPA). Demographic characteristics that were associated with LTPA and the environment score were included as confounders in regression models. Analyses were conducted in 2010 and included adjustment of the standard errors for the effects of clustering within the 80 areas (the unit of recruitment).

\section{Results}

More active women were younger, had lower BMI values, had higher levels of education, were employed full-time, were not married, had no children, were Australian-born, were non-smokers, did not have an illness/injury/disability preventing physical activity, and were a healthy weight (Table 1). Overall, women reported a median of 2 hours per week LTPA (Table 2). LTPA was significantly higher across increasing environment score categories and across increasing psychosocial score categories.

\begin{tabular}{|c|c|c|c|}
\hline $\begin{array}{l}\text { Leisure time } \\
\text { physical activity }\end{array}$ & n (\%) & Mean (SD) & Median (IQR) \\
\hline Overall & 3765 (100\%) & $206.3(272.9)$ & $120(0,280)$ \\
\hline \multicolumn{4}{|l|}{ Environment score } \\
\hline Low & $1064(28.3)$ & $181.2(269.7)$ & $90(0,240)$ \\
\hline Medium & 1159 (30.8) & $204.7(270.5)$ & $120(0,270)$ \\
\hline High & $1542(41.0)$ & $224.9(275.5)$ & $150(30,300)$ \\
\hline \multicolumn{3}{|c|}{$p_{\text {trend }}$} & $<.001$ \\
\hline \multicolumn{4}{|l|}{ Psychosocial score } \\
\hline Low & $1126(29.9)$ & $81.8(175.1)$ & $0(0,90)$ \\
\hline Medium & $1131(30.0)$ & $174.7(238.7)$ & $90(0,240)$ \\
\hline High & $1508(40.1)$ & $323.0(307.2)$ & $240(120,420)$ \\
\hline \multicolumn{3}{|c|}{$p_{\text {trend }}$} & $<.001$ \\
\hline
\end{tabular}

$S D$ standard deviation; IQR inter-quartile range; $R E A D /$ resilience for eating and inactivity despite inequality study.

* $p$-values from Cuzick non-parametric test for trend comparing leisure time physical activity values across increasing categories.
Higher environment factor scores were associated with increasing odds of higher levels of LTPA (Table 3). Compared with those with low environment scores, those with medium and high environment scores had 38\% and 67\% greater odds respectively of doing any LTPA (compared with no LTPA), $37 \%$ and $70 \%$ greater odds respectively of $\geq 120$ minutes/week LTPA (compared with $<120$ minutes/week LTPA), and $20 \%$ and $44 \%$ higher odds respectively of $\geq 280$ minutes/week LTPA (compared with $<280$ minutes/week LTPA).

When stratified by psychosocial score category, the association between the environment score and LTPA was largely attenuated except among women with low psychosocial scores (Table 4). Among these women, compared with those with low environment scores, women with medium or high environment scores had $76 \%$ (medium environment score) and 58\% (high environment score) greater odds of $\geq 120$ minutes/week of LTPA (compared with $<120$ minutes/week LTPA).

\section{Discussion}

This study examined associations between perceptions of the physical environment and LTPA among socioeconomically disadvantaged women possessing varying levels of individual and social factors known to be associated with physical activity. The findings suggest that a physical environment perceived to be supportive was associated with moderate levels of LTPA amongst women with psychosocial characteristics considered less beneficial for physical activity. This relationship was not evident among women with more favourable psychosocial characteristics, suggesting that perceiving a supportive physical environment may be particularly important for women with less favourable psychosocial characteristics.

Among women with low psychosocial scores, the perceived physical environment was associated with moderate amounts of LTPA (120 minutes/week) but not with any (versus none) or high LTPA ( $\geq 280$ minutes/week). This could be due to the categorisation of LTPA and the individual, social and environmental factors, which may have reduced precision to detect associations, although descriptive analyses identified associations in the expected directions. Alternately, these women may undertake activities that can be performed in their local neighbourhood (such as walking), and as a result are more aware of the characteristics of their physical environment. More active women may perform different types of physical activity (e.g. organised sport, or walking, running or cycling longer distances) undertaken outside the neighbourhood, reducing their awareness of local neighbourhood characteristics. This is plausible as for women reporting 120-279 minutes/week LTPA, a greater proportion comprised walking (62\%) compared with women reporting $\geq 280$ minutes/week (53\%). Another Australian study also 
Table $3 \mathrm{ORs}^{\mathrm{a}}$ for increasing LTPA by environment score and psychosocial score, among women in the READI study

\begin{tabular}{|c|c|c|c|}
\hline & \multicolumn{3}{|l|}{ LTPA } \\
\hline & $\begin{array}{l}\geq 1 \mathrm{~min} / \mathrm{wk} \\
\text { (vs. } 0 \mathrm{~min} / \mathrm{wk} \text { ) }\end{array}$ & $\begin{array}{l}\geq 120 \mathrm{mins} / \mathrm{wk} \\
\text { (vs. }<120 \mathrm{mins} / \mathrm{wk} \text { ) }\end{array}$ & $\begin{array}{l}\geq 280 \mathrm{mins} / \mathrm{wk} \\
(\mathrm{vs} .<280 \mathrm{mins} / \mathrm{wk})\end{array}$ \\
\hline \multicolumn{4}{|l|}{ Environment score } \\
\hline Low $(n=1009)$ & 1.0 (reference) & 1.0 (reference) & 1.0 (reference) \\
\hline Med $(n=1112)$ & $1.38(1.151 .64)^{* *}$ & $1.37(1.18,1.59)^{* *}$ & $1.20(1.01,1.43)^{*}$ \\
\hline High $(n=1495)$ & $1.67(1.40,2.00)^{* *}$ & $1.70(1.46,1.98)^{* *}$ & $1.44(1.22,1.70)^{* *}$ \\
\hline$p_{\text {trend }}$ & $<0.001$ & $<0.001$ & $<0.001$ \\
\hline \multicolumn{4}{|l|}{ Psychosocial score } \\
\hline Low $(n=1075)$ & 1.0 (reference) & 1.0 (reference) & 1.0 (reference) \\
\hline Medium $(n=1088)$ & $3.52(2.89,4.30)^{* * *}$ & $2.81(2.36,3.34)^{* * *}$ & $2.72(2.05,3.61)^{* * *}$ \\
\hline High $(n=1453)$ & $15.31(12.42,18.88)^{* * *}$ & $11.32(9.55,13.42)^{* * *}$ & $8.20(6.42,10.49)^{* * *}$ \\
\hline$p_{\text {trend }}$ & $<0.001$ & $<0.001$ & $<0.001$ \\
\hline
\end{tabular}

LTPA leisure time physical activity, READI resilience for eating and inactivity despite inequality study; ${ }^{*} \mathrm{p}<0.05{ }^{* *} \mathrm{p}<0.01{ }^{* * *} \mathrm{p}<0.001$.

a Odds ratios and $95 \%$ confidence intervals from generalised ordered logistic regression model adjusted for age, number of children, country of birth employment status, marital status, smoking status, and injury/illness/disability; robust standard errors adjusting for clustering by neighbourhood (the unit of recruitment). Generalised ordered logistic regression is appropriate for the ordered but not evenly spaced outcome data, and compares each increasing category to all data below the cut-point for that category.

found a greater proportion of participants walked for recreation within their neighbourhood than outside the neighbourhood, and that recreational walking within the neighbourhood was of greater duration than recreational walking outside the neighbourhood [27]. This suggests that amongst women with low psychosocial scores, perceiving a supportive environment may be important for achieving levels of LTPA that approximate the recommended 150 minutes/week [28,29]. Plausibly, women who have high psychosocial scores may be active irrespective of their environment, whereas for women with lower psychosocial scores, perceiving a supportive environment may be necessary to influence LTPA.

This study is limited by its cross-sectional design. It is plausible that active women perceive their environments as more supportive of activity or are more aware of their physical environment, or that women with more favourable psychosocial characteristics (e.g. higher physical activity self-efficacy) may over-optimistically report physical activity. Self-reported measures of LTPA and

Table 4 OR $^{\mathrm{a}}$ for LTPA according to environment score, stratified by psychosocial score, among women in the READI study

\begin{tabular}{|c|c|c|c|}
\hline \multirow{3}{*}{$\begin{array}{l}\text { Environment score by } \\
\text { psychosocial score }\end{array}$} & \multicolumn{3}{|l|}{ LTPA } \\
\hline & $\geq 1 \mathrm{~min} / \mathrm{wk}$ (vs. $0 \mathrm{~min} / \mathrm{wk}$ ) & $\geq 120$ mins/wk (vs. $<120$ mins/wk) & $\geq 280 \mathrm{mins} / \mathrm{wk}$ (vs. $<280 \mathrm{mins} / \mathrm{wk}$ ) \\
\hline & OR $(95 \% \mathrm{Cl})$ & OR $(95 \% \mathrm{Cl})$ & OR $(95 \% \mathrm{Cl})$ \\
\hline \multicolumn{4}{|l|}{ Low psychosocial score } \\
\hline Low environment score $(n=402)$ & 1.0 (reference) & 1.0 (reference) & 1.0 (reference) \\
\hline Med environment score $(n=350)$ & $1.21(0.88,1.65)$ & $1.76(1.22,2.54)^{*}$ & $1.34(0.75,2.37)$ \\
\hline High environment score $(n=323)$ & $1.19(0.87,1.65)$ & $1.58(1.07,2.34)^{*}$ & $1.38(0.80,2.40)$ \\
\hline \multicolumn{4}{|l|}{ Med psychosocial score } \\
\hline Low environment score $(n=316)$ & 1.0 (reference) & 1.0 (reference) & 1.0 (reference) \\
\hline Med environment score $(n=342)$ & $1.14(0.80,1.64)$ & $0.92(0.70,1.22)$ & $0.78(0.54,1.14)$ \\
\hline High environment score $(n=430)$ & $0.94(0.66,1.32)$ & $0.91(0.67,1.23)$ & $0.79(0.54,1.16)$ \\
\hline \multicolumn{4}{|l|}{ High psychosocial score } \\
\hline Low environment score $(n=291)$ & 1.0 (reference) & 1.0 (reference) & 1.0 (reference) \\
\hline Med environment score $(n=420)$ & $1.29(0.76,2.20)$ & $1.08(0.76,1.54)$ & $1.11(0.80,1.54)$ \\
\hline High environment score $(n=742)$ & $1.24(0.73,2.10)$ & $1.14(0.81,1.60)$ & $1.10(0.81,1.47)$ \\
\hline
\end{tabular}

LTPA leisure time physical activity, READI resilience for eating and inactivity despite inequality; ${ }^{*} \mathrm{p}<0.05$.

${ }^{a}$ Odds ratios and $95 \%$ confidence intervals from generalised ordered logistic regression model adjusted for age, number of children, country of birth,

employment status, marital status, smoking status, and injury/illness/disability; robust standard errors adjusting for clustering by neighbourhood (the unit of recruitment). Generalised ordered logistic regression is appropriate for the ordered but not evenly spaced outcome data, and compares each increasing category to all data below the cut-point for that category. 
environments may limit findings. However, there was variability within psychosocial and environmental score categories, and associations were observed with wellestablished correlates (e.g. age, BMI, education, employment status, number of children) [2] in expected directions. While a reliable and valid measure of physical activity was used (the IPAQ-L) [17], the psychometric properties of specific domains of activity such as during leisure time, have not been assessed. Lack of specificity in the environmental measures in relation to LTPA might have reduced the ability to detect associations [18]. For instance, the neighbourhood 'physical activity environment' refers mostly to walking activities, but the measure of LTPA is not limited to walking. However, walking is the most common leisure activity amongst Australian women [30] and more than $60 \%$ of participants reported walking for leisure. The large number of comparisons may be considered a limitation. However, by reducing the likelihood of false positives (a type I error) by adjusting for multiple comparisons, the likelihood of false negatives (a type II error) is increased, offering no real improvement [31].

While lack of access to a motor vehicle may have impacted the ability to access other more supportive environments further afield, only $6 \%$ of participants reported not having access to a motor vehicle (car ownership is high in Australia, with approximately 740 registered passenger vehicles per 1000 residents in Victoria [32]) access to a motor vehicle did not meet the definition of a confounder. We did not adjust for income, but previous analyses of data from this study found no association between income and LTPA [33], and hence income did not meet our definition of a confounder and was not considered as a potential covariate. It is plausible that there was residual confounding from the use of self-reported measures, unmeasured variables, or variables not included in our analyses.

This study was novel in its approach to understanding the complex relations between perceived individual, social and environmental factors and LTPA. While previous research has examined associations between individual, social and environmental factors and physical activity $[8,12,13]$, no studies have examined the effect on physical activity when there are 'mismatches' in levels of individual, social and environmental characteristics. Other strengths include the large sample size which enabled assessment of a range of factors simultaneously while adjusting for known covariates and stratification by variables of interest, appropriate statistical adjustment for the multilevel data structure, and the use of a theoretical framework to guide selection of correlates. Our work focused on an under-studied population group at high risk for inactivity and chronic disease, and provides insights for targeting and development of interventions to promote LTPA among women living in socioeconomically disadvantaged areas.

\section{Conclusion}

In conclusion, the findings suggest that for women with less favourable psychosocial characteristics (e.g. lower selfefficacy for physical activity, fewer intentions to be active, poorer social support networks), improving perceptions of the physical environment may be a worthwhile avenue for public health initiatives to increase physical activity. The findings also suggest that perceiving a supportive physical environment may make little difference to the likelihood of physical activity levels among women who already possess more favourable psychosocial characteristics (e.g. high self-efficacy for physical activity, greater intentions to be active, better social support networks). Strategies focused on improving general perceptions of the physical environment (e.g. promotion of facilities) may be warranted.

\section{Abbreviations}

Cl: Confidence interval; LTPA: Leisure-time physical activity; OR: Odds ratio; READI: Resilience for eating and inactivity despite inequality.

\section{Competing interests}

The authors declare that they have no competing interests.

\section{Authors' contributions}

VC conceptualised the research question, conducted the analyses and drafted the manuscript. KB made substantial contributions to the conception and design of the study and research question, interpretation of data, and critical revision of the manuscript. DC made substantial contributions to the conception and design of the study, interpretation of data, and critical revision of the manuscript. All authors read and approved the final manuscript.

\section{Acknowledgements}

This study was funded by an Australian National Health and Medical Research Council Strategic Award (ID 374241). The authors gratefully acknowledge the contributions of the Project Manager Dr Michelle Jackson, and the study participants. VC is supported by a NHMRC Public Health Postdoctoral Training Fellowship (ID 533917). DC is supported by a Public Health Research Fellowship from the Victorian Health Promotion Foundation. KB is supported by a NHMRC Senior Research Fellowship (ID 479513).

\section{Author details}

${ }^{1}$ Centre for Physical Activity \& Nutrition Research, Deakin University, 221 Burwood Hwy, Burwood, Victoria 3125, Australia. ${ }^{2}$ Current address: Menzies Research Institute Tasmania, Private Bag 23, Hobart, Tasmania, 7001, Australia.

Received: 14 August 2012 Accepted: 13 March 2013

Published: 27 March 2013

\section{References}

1. Gidlow C, Johnston LH, Crone D, Ellis N, James D: A systematic review of the relationship between socio-economic position and physical activity. Health Educ J 2006, 65:338-367.

2. Trost SG, Owen N, Bauman AE, Sallis JF, Brown W: Correlates of adults' participation in physical activity: review and update. Med Sci Sports Exerc 2002, 34(12):1996-2001.

3. Beenackers MA, Kamphuis CB, Giskes K, Brug J, Kunst AE, Burdorf A, van Lenthe FJ: Socioeconomic inequalities in occupational, leisure-time, and transport related physical activity among European adults: a systematic review. Int J Behav Nutr Phys Act 2012, 9:116.

4. Sallis J, Owen N: Ecological models of health behavior. In Health Behavior and Health Education: Theory, Research \& Practice. 3rd edition. Edited by Glanz K, Rimer BK, Lewis FM. San Francisco, USA: Jossey-Bass; 2002:462-484. 
5. Stokols D: Establishing and maintaining healthy environments. Toward a social ecology of health promotion. Am Psychol 1992, 47(1):6-22.

6. Ball K, Timperio A, Salmon J, Giles-Corti B, Roberts R, Crawford D: Personal, social and environmental determinants of educational inequalities in walking: a multilevel study. J Epidemiol Community Health 2007, 61(2):108-114.

7. Cleland VJ, Ball K, Salmon J, Timperio AF, Crawford DA: Personal, social and environmental correlates of resilience to physical inactivity among women from socio-economically disadvantaged backgrounds. Health Educ Res 2010, 25(2):268-281.

8. Cleland V, Ball K, Hume C, Timperio A, King AC, Crawford D: Individual, social and environmental correlates of physical activity among women living in socioeconomically disadvantaged neighbourhoods. Soc Sci Med 2010, 70(12):2011-2018.

9. Bauman A, Bull FC: Environmental correlates of physical activity and walking in adults and children: A review of reviews. London: National Institute of Health and Clinical Excellence; 2007.

10. Plotnikoff RC, Pickering MA, Rhodes RE, Courneya KS, Spence JC: A test of cognitive mediation in a 12-month physical activity workplace intervention: does it explain behaviour change in women? Int I Behav Nutr Phys Act 2010, 7:32.

11. Fjeldsoe BS, Miller YD, Marshall AL: Social cognitive mediators of the effect of the MobileMums intervention on physical activity. Health Psychol 2012. in press.

12. Giles-Corti B, Donovan RJ: The relative influence of individual, social and physical environment determinants of physical activity. Soc Sci Med 2002, 54(12):1793-1812.

13. Pan SY, Cameron C, Desmeules M, Morrison H, Craig CL, Jiang X: Individual, social, environmental, and physical environmental correlates with physical activity among Canadians: a cross-sectional study. BMC Publ Health 2009, 9:21.

14. Cleland VJ, Hume C, Crawford D, Timperio A, Hesketh K, Baur L, Welch N, Salmon J, Ball K: Urban-rural comparison of weight status among women and children living in socioeconomically disadvantaged neighbourhoods. Med J Aust 2010, 192(3):137-140.

15. MacFarlane AM, Abbott GR, Crawford DA, Ball K: Sociodemographic and behavioural correlates of weight status among women with children living in socioeconomically disadvantaged neighbourhoods. Int J Obes (Lond) 2009, 33(11):1289-1298.

16. McLennan W: 1996 Census of population and housing: socioeconomic index for areas. In. Australian Bureau of Statistics: Canberra; 1998.

17. Craig CL, Marshall AL, Sjostrom M, Bauman AE, Booth ML, Ainsworth BE, Pratt M, Ekelund U, Yngve A, Sallis JF, et al: International physical activity questionnaire: 12-country reliability and validity. Med Sci Sports Exerc 2003, 35(8):1381-1395.

18. Giles-Corti B, Timperio A, Bull F, Pikora T: Understanding physical activity environmental correlates: increased specificity for ecological models. Exerc Sport Sci Rev 2005, 33(4):175-181.

19. Sharpe PA, Granner ML, Hutto BE, Wilcox S, Peck L, Addy CL: Correlates of physical activity among African American and white women. Am J Health Behav 2008, 32(6):701-713.

20. Marcus BH, Selby VC, Niaura RS, Rossi JS: Self-efficacy and the stages of exercise behavior change. Res Q Exerc Sport 1992, 63(1):60-66.

21. Kendzierski D, DeCarlo KJ: Physical activity enjoyment scale: two validation studies. J Sport Exerc Psychol 1991, 13:50-64.

22. Giles-Corti B, Donovan RJ: Relative influences of individual, social environmental, and physical environmental correlates of walking. Am J Public Health 2003, 93(9):1583-1589.

23. Sallis JF, Grossman RM, Pinski RB, Patterson TL, Nader PR: The development of scales to measure social support for diet and exercise behaviors. Prev Med 1987, 16(6):825-836

24. Mujahid MS, Diez Roux AV, Morenoff JD, Raghunathan T: Assessing the measurement properties of neighborhood scales: from psychometrics to ecometrics. Am J Epidemiol 2007, 165(8):858-867.

25. World Health Organization: Obesity: preventing and managing the global epidemic. Geneva: World Health Organization; 2000.

26. Williams R: Generalized ordered logit/partial proportional-odds models for ordinal dependent variables. Stata J 2006, 6:58-82

27. Giles-Corti B, Knuiman M, Timperio A, Van Niel K, Pikora TJ, Bull FC, Shilton $\mathrm{T}$, Bulsara M: Evaluation of the implementation of a state government community design policy aimed at increasing local walking: design issues and baseline results from RESIDE, Perth Western Australia. Prev Med 2008, 46(1):46-54
28. Australian Department of Health and Aged Care: National physical activity guidelines for Australians. Canberra: Department of Health and Aged Care; 1999:1-2.

29. Haskell WL, Lee IM, Pate RR, Powell KE, Blair SN, Franklin BA, Macera CA, Heath GW, Thompson PD, Bauman A: Physical activity and public health: updated recommendation for adults from the american college of sports medicine and the american heart association. Med Sci Sports Exerc 2007, 39(8):1423-1434

30. Australian Sports Commission: Participation in Exercise, Recreation and Sport: Annual Report 2009. Canberra: Australian Sports Commission; 2009.

31. Rothman KJ: Modern Epidemiology. Boston: Little, Brown \& Co.; 1986.

32. Australian Bureau of Statistics: Motor Vehicle Census (cat. no. 9309.0). Canberra: ABS; 2009

33. Cleland V, Ball K, Crawford D: Socioeconomic position and physical activity among women in Melbourne, Australia: does the use of different socioeconomic indicators matter? Soc Sci Med 2012, 74(10):1578-1583.

doi:10.1186/1471-2458-13-280

Cite this article as: Cleland et al.: Is a perceived supportive physical environment important for self-reported leisure time physical activity among socioeconomically disadvantaged women with poor psychosocial characteristics? An observational study. BMC Public Health 2013 13:280.

\section{Submit your next manuscript to BioMed Central and take full advantage of:}

- Convenient online submission

- Thorough peer review

- No space constraints or color figure charges

- Immediate publication on acceptance

- Inclusion in PubMed, CAS, Scopus and Google Scholar

- Research which is freely available for redistribution

Submit your manuscript at www.biomedcentral.com/submit
C Biomed Central 